\title{
Rayleigh-Bénard convection in non-Newtonian Carreau fluids with arbitrary conducting boundaries
}

\author{
Mondher Bouteraa, Chérif Nouar* \\ LEMTA UMR 7563 CNRS - Université de Lorraine \\ ENSEM, 2 Avenue de la Forêt de Haye, TSA 60604 - 54516 Vandoeuvre lès Nancy cedex, \\ France
}

\begin{abstract}
The objective of the present work is to investigate the Rayleigh-Bénard convection in non-Newtonian fluids with arbitrary conducting boundaries. A linear and weakly nonlinear analysis is performed. The rheological behavior of the fluid is described by the Carreau model. As a first step, the critical Rayleigh number and wavenumber for the onset of convection are computed as a function of the ratios $\xi^{b}$ and $\xi^{t}$ of the thermal conductivities of the bottom and top slabs to that of the fluid. In the second step, the preferred convection pattern is determined using an amplitude equation approach. The stability of rolls and squares is investigated as a function of $\left(\xi^{b}, \xi^{t}\right)$ and the rheological parameters. The bounded region of $\left(\xi^{b}, \xi^{t}\right)$ space where squares are stable decreases with increasing shear-thinning effects. This is related to the fact that shear-thinning effects increase the nonlinear interactions between sets of rolls that constitute the square patterns [1]. For a significant deviation from the critical conditions, the nonlinear convection terms and nonlinear viscous terms become stronger, reducing overall the stability domain of squares. The largest Nusselt number, $N u$, is obtained for perfectly conducting boundaries. For a given $\left(\xi^{b}, \xi^{t}\right)$, the stable solution yields the largest Nusselt number. The enhancement of heat transfer due to shear-thinning effects is significantly reduced for poorly heat conducting plates.
\end{abstract}

\footnotetext{
* Corresponding author.

Email address: cherif.nouar@univ-lorraine.fr (Chérif Nouar)
} 
Keywords: Rayleigh-Bénard convection, non-Newtonian fluid, shear-thinning fluid, pattern selection

\section{Introduction}

The problem of Rayleigh-Bénard convection (RBC) in Newtonian and nonNewtonian fluids layer heated from below and cooled from above remains one of the classical problems of fluid dynamics and heat transfer. In spite of intensive 5 studies made in the past and extensive research work undertaken so far to understand the competition between convective structures (rolls, squares and hexagons) which are often influenced by the boundary conditions (see Holmedal et al. [2]; Clever and Busse [3]) and other parameters such as temperaturedependence of viscosity (see White [4]; Palm [5]; Richter [6]; Olivier and Booker [7]; Busse and Frick [8]; Jenkins [9] ), there are still many outstanding issues that need to be answered.

May be one of the most important question to be addressed is the effect of conductive horizontal plates on the heat transfer and the convection patterns. For instance, in geophysical problems and particularly in the context 15 of the Earth's mantle convection, continents and oceans impose different thermal boundary conditions at the top of the mantle: continents act as insulators while a fixed temperature is imposed by oceans. These different thermal boundary conditions affect the convective flow and the heat transport in the Earth's mantle[10].

20 Actually, in most numerical investigations of RBC, the plates are assumed to be infinitely heat conducting, and a fixed temperature at the boundaries is imposed, while in engineering and geophysical problems as well as in laboratory experiments the boundaries have a finite conductivity. This may lead to a discrepancy between the experimental and the numerical/theoretical results. The

25 ratios $\xi^{b}$ and $\xi^{t}$ between the thermal conductivities of the bottom and top slabs and that of the fluid may have a significant effect and must be taken into account as additional parameters [11]. 
The influence of the thermal conductivity of the boundaries on Rayleigh-Bénard convection was first investigated in the Newtonian case by Busse and Riahi 12] using a weakly nonlinear analysis. They considered the situation where $\xi^{b}=\xi^{t}=\xi<<1$ and found that the wavelength of convection flow becomes very large in comparison with the height of the layer and only square patterns are stable. This result was confirmed and extended to the fully nonlinear problem by Proctor [13] using a 'shallow water theory'. Afterwards, Jenkins and

35 Proctor [14] determined the critical value of the thermal conductivities ratios $\xi^{b}=\xi^{t}=\xi_{c}$ at which the preferred planform changes from square cell to roll. For $\operatorname{Pr}>10$, they found that the preferred planform is rolls when $\xi>1$, and squares when $\xi<1$. Le Gal et al. [15] carried out experiments to study Rayleigh-Bénard convection in silicone oil confined between two glass plates. So

40 that $\xi^{b}=\xi^{t}=\xi=7$. Near the threshold of convective instability, at $\epsilon<0.021$, where $\epsilon$ is the relative distance from the onset of instability, they observed cells of square planforms. But when $0.024<\epsilon<0.057$, the amplitude of two mutually perpendicular roll sets underwent periodic oscillations in antiphase with another; as $\epsilon$ was increased and convection became more intense, one set became predominant and then only roll still stable. This experiment was subsequently modified by Le Gal and Croquette [16] : glass was replaced by plexiglass and water was used as the working fluid, so that $\xi=0.4$. In contrast to the preceding experiment, squares were observed in a wide range of $\epsilon$ values without any signs of destabilization. The authors think that in the first case, the silicone oil behaves as a mixture and the observed features were governed by the thermophoresis .

Although extensive studies have been devoted to understand the influence of the thermal boundary conditions on the Rayleigh-Bénard convection in Newtonian fluids, only a limited number of works have dealt with complex fluids.

${ }_{55}$ In comparison with the Newtonian system, the nonlinearity of the rheological law introduces an additional coupling in the velocity component. Recently, Bouteraa and Nouar [17] have investigated the influence of shear-thinning effects on the convection in a horizontal layer of a shear-thinning fluid between 
two horizontal symmetric plates of finite thermal conductivity. The rheological behavior of the fluid is described by the Carreau model. The authors found that: (i) the characteristic time of instability $\tau_{0}$ increases significantly when $\xi<1$, (ii) the critical value of the shear-thinning degree $\alpha_{c}$ above which the bifurcation becomes subcritical increases with decreasing $\xi$, and (iii) the critical value $\xi_{c}$ at which the planform changes from square-cell solution $\left(\xi<\xi_{c}\right)$ to two-dimensional roll solution $\left(\xi>\xi_{c}\right)$ decreases with increasing shear-thinning effects.

In some experimental situations, $\xi^{t} \neq \xi^{b}$. For Newtonian fluids, Riahi[11] [18] has studied this problem and demonstrated, using a linear stability analysis of stationary flows the enormous influence of thermal boundary conditions (when 70 $\left.\xi^{b} \neq \xi^{t}\right)$ on the competition between the convection patterns. He found that squares are stable when rolls are unstable and vice versa, and always hexagonal patterns are unstable. No hysteresis effect is found. In addition, Riahi 18] has also shown that square planforms are preferred in a bounded region $\Omega$ in the $\left(\xi^{b}, \xi^{t}\right)$-space coordinate system and rolls are favored only outside $\Omega$.

75 When $\operatorname{Pr}<0.025$, the region $\Omega$ is quite small and disappears as $\operatorname{Pr}=0$. However, for $\operatorname{Pr}>7, \Omega$ is largest and nearly independent of $\operatorname{Pr}$. Using nonlinear developments, Clever and Busse [19] [3] demonstrated in the case of stressfree nearly insulating top plate and highly conducting no-slip lower plate, that two-dimensional rolls are stable near the onset, but become unstable at higher so Rayleigh number and are replaced by which is called hexharoll convection. From experimental point of view, Darbouli et al. 20] have investigated RayleighBénard convection for viscoplastic fluids confined in a cylindrical cell. They used two different horizontal plates of finite thermal conductivity. The bottom and upper walls are made respectively of copper alloy and glass. They used distilled 85 water as Newtonian fluid to validate their experimental setup and an aqueous solution of Carbopol 940 as viscoplastic fluid. In these situations, the ratios $\xi^{t}$ and $\xi^{b}$ are estimated to $\xi^{t}=2$ and $\xi^{b}=201.6$ for both fluids (authors estimated that the solution of Carbopol 940 has the same thermal conductivity than water). Hence, it is no longer possible to rely on the assumption that the plates 
90 are held at fixed and uniform temperatures, which corresponds to plates with infinite thermal conductivity.

The purpose of the present work is to study the influence of arbitrary thermal-conducting top and bottom boundaries on nonlinear processes of Rayleigh Bénard convection, and to see the influence of the shear-thinning effect on the preferred flow pattern. The finite conductivity of the slabs remains one explanation for differences between results obtained in experiments and numerical investigations. We hope that our findings will shed new light on the interpretation of the results obtained by Darbouli et al. [20] although the fluid used is not only shear-thinning but has also a yield stress.

\section{Physical and mathematical model}

\subsection{General equations and parameters}

We consider a horizontal layer of a shear-thinning fluid of height $\hat{d}$ confined between two horizontal plates that are infinite in extent and which have a thickness $\Lambda \hat{d}$, where $\Lambda$ is of order unity. The outer surface of the bottom and top plates are kept at constant temperatures respectively $\hat{T}_{0}+\Delta \hat{T} / 2$ and $\hat{T}_{0}-\Delta \hat{T} / 2$, with $\Delta \hat{T}>0$. The fluid has density $\hat{\rho}$, thermal conductivity $\hat{k}$, thermal coefficient expansion (at constant pressure) $\hat{\beta}$ and viscosity $\hat{\mu}_{0}$ at zero shear rate. The top slab has a thermal conductivity $\hat{k}_{p}^{t}$ and a thermal diffusivity $\hat{\kappa}_{p}^{t}$. ${ }_{110}$ The corresponding quantities for the bottom slab are denoted $\hat{k}_{p}^{b}$ and $\hat{\kappa}_{p}^{b}$. Here and in what follows, $(t)$ and $(b)$ refer to the top and bottom and the quantities with hat $(\hat{.})$ are dimensional. Because of the thermal expansion, the temperature difference between the two plates, induces a vertical density stratification. Heavy cold fluid is above a light warm fluid. For small $\Delta \hat{T}$, the fluid remains motionless and the heat is transferred by conduction, with a linear temperature profile across the fluid layer.

In the fluid, $0<\hat{z}<\hat{d}$, the hydrostatic solution and the temperature profile 
are:

$\frac{d \hat{P}}{d \hat{z}}=-\hat{\rho}_{0} \hat{g}\left[1-\hat{\beta}\left(\hat{T}-\hat{T}_{0}\right)\right] \quad$ and $\quad \hat{T}_{\text {cond }}=\hat{T}_{0}+\frac{\Delta \hat{T}}{1+\Lambda / \xi^{(b)}+\Lambda / \xi^{(t)}}\left[\frac{1}{2}-\frac{\hat{z}}{\hat{d}}\right]$

where, $\hat{g}$ is the acceleration due to gravity. Here, the z-axis is directed upwards, with the origin located at the bottom plate. The reference temperature $\hat{T}_{0}$ is the temperature in the middle of the fluid layer and $\hat{\rho}_{0}$ is the fluid density at $\hat{T}_{0}$. Here, $\hat{T}_{0}=\hat{T}_{1}-\left(1 / 2+\Lambda / \xi^{b}\right) \Delta \hat{T}_{f}$, where $\hat{T}_{1}$ is the temperature on the outer surface of the bottom plate and $\Delta \hat{T}_{f}$ the temperature difference between the top and the bottom of the fluid layer: $\Delta \hat{T}_{f}=\Delta \hat{T} /\left(1+\Lambda / \xi^{t}+\Lambda / \xi^{(b)}\right)$.

${ }_{125}$ The temperature profile in the top and bottom plates are:

$$
\begin{aligned}
\hat{T}_{\text {cond }}= & \hat{T}_{0}+\frac{\Delta \hat{T}}{\xi^{t}+\Lambda\left(1+\xi^{t} / \xi^{b}\right)}\left[1+\frac{\Lambda}{2}\left(1-\frac{\xi^{t}}{\xi^{b}}\right)-\frac{1}{2} \xi^{t}-\frac{\hat{z}}{\hat{d}}\right], \\
& \hat{d} \leq \hat{z} \leq(1+\Lambda) \hat{d}
\end{aligned}
$$

and

$$
\hat{T}_{\text {cond }}=\hat{T}_{0}+\frac{\Delta \hat{T}}{\xi^{b}+\Lambda\left(1+\xi^{b} / \xi^{t}\right)}\left[\frac{1}{2} \xi^{b}-\frac{\Lambda}{2}\left(1-\xi^{b} / \xi^{t}\right)-\frac{\hat{z}}{\hat{d}}\right], \quad-\Lambda \hat{d} \leq \hat{z} \leq 0 .
$$

When the bottom and top plates are poor thermal conductors, a large part of $\Delta \hat{T}$ occurs across the plates, and remains only a small part $\Delta \hat{T}_{f}$ of $\Delta \hat{T}$, acting as the driving force for the convection. When $\Delta \hat{T}_{f}$, exceeds a critical value, the convection sets in and a so-called convective patterns emerge. The stability of the hydrostatic solution is considered by introducing temperature and pressure perturbation as well as a fluid motion. Boussinesq approximation is adopted, i.e., the temperature dependence of the fluid properties can be neglected except for the temperature induced density difference in the buoyant force that drives the flow. The heat production due to viscosity is neglected. Distances are scaled with $\hat{d}$, velocity with $\hat{\kappa} / \hat{d}$, where $\hat{\kappa}$ is the thermal diffusivity of the fluid, time with $\hat{d}^{2} / \hat{\kappa}$, temperature with $\Delta \hat{T}_{f}$, pressure and stresses with $\hat{\kappa} \hat{\mu}_{0} / \hat{d}^{2}$. Using these scales, the dimensionless perturbation equations read:

$$
\begin{aligned}
\frac{\partial \boldsymbol{\nabla}^{2} w}{\partial t}-\boldsymbol{e}_{z} \cdot[\boldsymbol{\nabla} \times \boldsymbol{\nabla} \times[(\boldsymbol{u} . \boldsymbol{\nabla}) \boldsymbol{u}]]= & \operatorname{Pr} \Delta^{2} w+\operatorname{Ra} \operatorname{Pr} \boldsymbol{\nabla}_{H}^{2} \theta- \\
& \operatorname{Pr}[\boldsymbol{\nabla} \times \boldsymbol{\nabla} \times[\boldsymbol{\nabla} \cdot(\mu-1) \dot{\gamma}]] \cdot \boldsymbol{e}_{z}, \\
\frac{\partial \theta}{\partial t}+(\boldsymbol{u} \cdot \boldsymbol{\nabla}) \theta=w+\nabla^{2} \theta, &
\end{aligned}
$$


in the fluid, and

$$
\frac{\partial \tilde{\theta}}{\partial t}=\frac{\hat{\kappa}_{p}^{(b, t)}}{\hat{\kappa}} \nabla^{2} \tilde{\theta}
$$

in the bounding slabs. Here, $\boldsymbol{e}_{z}$ denotes the unit vector in the vertical direction, $w=\boldsymbol{u}(\boldsymbol{x}, t) \cdot \boldsymbol{e}_{z}$ is the vertical velocity field and $\theta(\boldsymbol{x}, t)$ represents the temperature deviations from their values in the conduction state. The temperature perturbation in the slabs is denoted $\tilde{\theta}(\boldsymbol{x}, t)$. The position vector $\boldsymbol{x}$ has components $x, y, z$. The pressure was eliminated by applying twice the rotation to the momentum balance equation. The state of the fluid is controlled by two dimensionless variables. These are the Rayleigh number $R a$ which measures the ratio between the driving buoyancy and the damping forces, and the Prandtl number $\operatorname{Pr}$ which represents the ratio between thermal and viscous diffusion times:

$$
R a=\frac{\hat{\rho}_{0} \hat{g} \hat{\beta} \Delta \hat{T}_{f} \hat{d}^{3}}{\hat{\kappa} \hat{\mu}_{0}} \quad ; \quad \operatorname{Pr}=\frac{\hat{\mu}_{0}}{\hat{\rho}_{0} \hat{\kappa}} .
$$

\subsection{Rheological model and parameters}

In the present study the behavior of the shear-thinning fluid is described by the Carreau model given by:

$$
\frac{\hat{\mu}-\hat{\mu}_{\infty}}{\hat{\mu}_{0}-\hat{\mu}_{\infty}}=\left(1+\hat{\lambda}^{2} \hat{\Gamma}\right)^{\frac{n_{c}-1}{2}} \quad \text { with } \quad \Gamma=\frac{1}{2} \dot{\gamma}_{i j} \dot{\gamma}_{i j} .
$$

Here, $\hat{\mu}_{0}$ and $\hat{\mu}_{\infty}$ are the viscosities at low and high shear rate, $\left(n_{c}<1\right)$ the power-law exponent characterizing the shear-thinning regime and $\hat{\lambda}$ the characteristic time of the fluid. The location of the transition from the Newtonian plateau to the shear-thinning regime is determined by $\hat{\lambda}$, since $1 / \hat{\lambda}$ defines the characteristic shear rate marking the onset of shear-thinning. The infinite shear viscosity, $\hat{\mu}_{\infty}$, is generally associated with a breakdown of the fluid, and is frequently significantly smaller $\left(10^{-3}\right.$ to $10^{-4}$ times smaller $)$ than $\hat{\mu}_{0}$, see Bird et al. [21] and Tanner [22]. The ratio $\hat{\mu}_{\infty} / \hat{\mu}_{0}$ will be thus neglected in the following. The dimensionless effective viscosity is then

$$
\mu=\frac{\hat{\mu}}{\hat{\mu}_{0}}=\left(1+\lambda^{2} \Gamma\right)^{\frac{n_{c}-1}{2}} \quad \text { with } \quad \lambda=\frac{\hat{\lambda}}{\hat{d}^{2} / \hat{\kappa}} .
$$


The Newtonian behavior, $\hat{\mu}=\hat{\mu}_{0}$, is obtained by setting $n_{c}=1$ or $\hat{\lambda}=0$. For a small amplitude disturbance, the viscosity can be expanded about the hydrostatic solution,

$$
\mu=1+\left(\frac{n_{c}-1}{2}\right) \lambda^{2} \Gamma+\frac{1}{2}\left(\frac{n_{c}-1}{2}\right)\left(\frac{n_{c}-3}{2}\right) \lambda^{4} \Gamma^{2}+\ldots
$$

165 At lowest nonlinear order, a relevant rheological parameter is the 'degree of shear-thinning'

$$
\alpha=\left|\frac{d \mu}{d \Gamma}\right|_{\Gamma=0}=\frac{1-n_{c}}{2} \lambda^{2} .
$$

\subsection{Boundary conditions}

For the perturbation velocity $w$, we imposed a realistic boundary conditions on the top and bottom plates: The slabs are rigid, thus enforcing the no-slip boundary conditions (NSBC), which implies

$$
w=D w=0 \quad \text { at } \quad z=0,1 .
$$

For thermal boundary conditions, a constant and uniform temperature is assumed on the outer surface of each plate

$$
\tilde{\theta}=0 \quad \text { at } \quad z=-\Lambda, 1+\Lambda
$$

The continuity condition of temperature and heat flux at the interface liquid/solid reads

$$
\begin{array}{rlc}
\theta=\tilde{\theta} \quad \text { at } & z=0,1, \\
D \theta=\xi^{(b, t)} D \tilde{\theta} \quad \text { at } & z=0,1 .
\end{array}
$$

${ }_{175}$ Where $D \equiv \frac{\partial}{\partial z}$ and $\xi^{(b, t)}=\frac{\hat{k}_{p}^{(b, t)}}{\hat{k}}$.

Remark: Following Holmedal et al. 2], applying the transformation

$$
\left(u, v, w, p, \theta, \tilde{\theta}^{b, t}, z\right) \longrightarrow\left(u, v,-w, p,-\theta,-\tilde{\theta}^{b, t}, 1-z\right)
$$

to Eqs. (44) - (6), (14) and (15), it can be shown that the problem is invariant if $\left(\xi^{b}, \xi^{t}\right) \longrightarrow\left(\xi^{t}, \xi^{b}\right)$. Therefore, the preferred convection pattern, its stability and the heat transfer is not modified if the bottom slab is replaced by the top 180 slab and vice versa. 


\section{Linear stability analysis}

\subsection{Critical conditions and critical modes}

In the linear theory, $\boldsymbol{u}$ and $\theta$ are assumed infinitesimal. The nonlinear terms in (4)-(5) can be neglected. We obtain :

$$
\begin{aligned}
& \frac{1}{\operatorname{Pr}} \frac{\partial \Delta w}{\partial t}=\Delta^{2} w+R a \Delta_{H} \theta, \\
& \frac{\partial \theta}{\partial t}=w+\Delta \theta, \\
& \frac{\partial \tilde{\theta}}{\partial t}=\frac{\hat{\kappa}_{p}^{(b, t)}}{\hat{\kappa}} \Delta \tilde{\theta} .
\end{aligned}
$$

At this stage, no non-Newtonian effects enter the problem. For equations (17)(19), we seek a normal mode solution

$$
\begin{aligned}
& w(x, y, z, t)=F_{11}(z) f(x, y) \exp (s t) \\
& \theta(x, y, z, t)=G_{11}(z) f(x, y) \exp (s t) \\
& \tilde{\theta}(x, y, z, t)=\tilde{G}_{11}(z) f(x, y) \exp (s t),
\end{aligned}
$$

where, $s=s_{r}+i s_{i}$ is a complex number and $f(x, y)$ satisfies the two-dimensional Helmoltz equation $\Delta_{H} f=-k^{2} f$. Here $k$ is the norm of the horizontal wavenumber $\boldsymbol{k}$. Substituting (20)-(22) into (17)-(19), leads to the differential equations

$$
\begin{aligned}
& s \operatorname{Pr}^{-1}\left(D^{2}-k^{2}\right) F_{11}=-k^{2} R a G_{11}+\left(D^{2}-k^{2}\right)^{2} F_{11}, \\
& s G_{11}=F_{11}+\left(D^{2}-k^{2}\right) G_{11}, \\
& s \tilde{G}_{11}=\frac{\hat{\kappa}_{p}^{(b, t)}}{\hat{\kappa}}\left(D^{2}-k^{2}\right) \tilde{G}_{11} .
\end{aligned}
$$

190 The boundary conditions are:

$$
\begin{array}{ll}
F_{11}=D F_{11}=0 & \text { at } \quad z=0,1 \\
\tilde{G}_{11}=0 & \text { at } \quad z=-\Lambda, 1+\Lambda, \\
G_{11}=\tilde{G}_{11} & \text { at } \quad z=0,1 \\
D G_{11}=\xi^{(b, t)} D \tilde{G}_{11} & \text { at } \quad z=0,1 .
\end{array}
$$


Denoting by the superscript * the complex conjugate, multiplying (23) by $F_{11}^{*}$, (24) by $-k^{2} R a G_{11}^{*}$, (25) by $-k^{2} R a\left(\hat{\kappa} / \hat{\kappa}^{b, t}\right) \xi^{(b, t)} \tilde{G}_{11}^{*}$, summing and integrating over the interval $[-\Lambda, 1+\Lambda]$, we have

$$
\begin{aligned}
& s\left[\int_{-\Lambda}^{1+\Lambda}\left(\left|D F_{11}\right|^{2}+k^{2}\left|F_{11}\right|^{2}+k^{2} R a\left|G_{11}\right|^{2}+k^{2} R a \xi^{(b, t)} \frac{\hat{\kappa}}{\hat{\kappa}_{p}^{(b, t)}}\left|\tilde{G}_{11}\right|^{2}\right) \mathrm{d} z\right]= \\
& k^{2} R a \int_{-\Lambda}^{1+\Lambda}\left(F_{11} G_{11}^{*}+F_{11}^{*} G_{11}\right) \mathrm{d} z-k^{2} R a \int_{-\Lambda}^{1+\Lambda}\left(\left|D G_{11}\right|^{2}+k^{2}\left|G_{11}\right|^{2}\right) \mathrm{d} z \quad(30) \\
& -k^{2} R a \xi^{(b, t)} \int_{-\Lambda}^{1+\Lambda}\left(\left|D \tilde{G}_{11}\right|^{2}+k^{2}\left|\tilde{G}_{11}\right|^{2}\right) \mathrm{d} z,
\end{aligned}
$$

with, $F_{11}=0$ as $z \in[-\Lambda, 0] \cup\left[1,1+\Lambda, G_{11}=0\right.$ as $z \in[-\Lambda, 0[\cup] 1,1+\Lambda]$ and $\tilde{G}_{11}=0$ when $\left.z \in\right] 0,1[$. The right hand side of equation (30) is real, therefore $s_{i}=0$ : the linearized equations (23) - 25) satisfy the principle of exchange of stability. The conduction state loses its stability to a convective stationary state in a smooth way.

The set of differential equations (23)-(25) is an eigenvalue problem where $s$ is the eigenvalue and $\boldsymbol{X}_{11}=\left(F_{11}, G_{11}, \tilde{G}_{11}\right)$ the eigenvector. It can be written

$$
s \boldsymbol{M} \cdot \boldsymbol{X}_{11}=\boldsymbol{L} \cdot \boldsymbol{X}_{11} .
$$

Actually, Eq. 25 can be solved analytically:

$$
\begin{aligned}
& \tilde{G}_{11}(z)=G_{11}(z=1) \frac{\sinh \left(\tilde{k}^{(t)}(1+\Lambda-z)\right)}{\sinh \left(\tilde{k}^{(t)} \Lambda\right)} \quad ; \quad 1 \leq z \leq 1+\Lambda, \\
& \tilde{G}_{11}(z)=G_{11}(z=0) \frac{\sinh \left(\tilde{k}^{(b)}(\Lambda+z)\right)}{\sinh \left(\tilde{k}^{(b)} \Lambda\right)} \quad ; \quad-\Lambda \leq z \leq 0,
\end{aligned}
$$

with $\tilde{k}^{(b, t)}=\sqrt{k^{2}+s \hat{\kappa} / \hat{\kappa}_{p}^{(b, t)}}$. Hence, the eigenvalue problem (23)-(25) can be restrained to the fluid domain, i.e. Eqs (23) - (24), with the boundary conditions

$$
D G_{11}= \pm \xi^{(b, t)} \tilde{k}^{(b, t)} G_{11} \operatorname{coth}\left(\tilde{k}^{(b, t)} \Lambda\right) \quad ; \quad z=0,1 .
$$

205 The eigenvalue problem (23)-(24) with the boundary conditions (34) is solved using a Chebyshev collocation method. By setting $s=0$, one obtains the 
marginal stability curve $R a(k)$. The minimum of this curve gives the critical Rayleigh number $R a_{c}$ and the corresponding wavenumber $k_{c} . R a_{c}$ allows us to determine the critical $\Delta \hat{T}_{f}$ at which the system changes from the hydrostatic state to the state of cellular motion and $k_{c}$ provides information about the horizontal periodicity of the patterns at the onset of convection. We recall that the transition from the rest state to the convective state is independent of the Prandtl number. Figure 1 displays the variation of $R a_{c}$ and $k_{c}$ as a function of the ratio $\xi^{b}$ for different values of $\xi^{t}$. The dimensionless thickness of the 215 plates is fixed at $\Lambda=1$. As expected, the critical conditions are the same if the upper plate is exchanged with the lower one. We recover the following asymptotic limits: (i) $R a_{c}=1707.7$ and $k_{c}=3.12$ for two perfectly conducting boundaries, (ii) $R a_{c}=720$ and $k_{c} \rightarrow 0$ for two insulating boundaries and (iii) $R a_{c}=1296$ and $k_{c}=2.55$ for one insulating boundary and the other one perfectly conducting. When either $\xi^{b}$ or $\xi^{t}$ or both are low, the wavelength of the convection becomes larger and the critical Rayleigh number $R a_{c}$ is reduced. Mathematically, the decrease of $R a_{c}$ is caused by the weakening of the thermal boundary conditions (28), (29) for the temperature fluctuations $\theta$ as $\xi^{b}$ or $\xi^{t}$ or both decrease from $10^{3}$ to $10^{-3}$. These results are in very good quantitative agreement with those obtained by Sparrow et al. 23], Proctor [13], Jenkins and Proctor [14], Carriere et al. 24] and Cerisier et al. 25]. From a physical point of view, it means that when a thermal fluctuations occurs in the fluid close to infinitely conducting boundaries, it easily relaxes. However, when either $\xi^{b}$ or $\xi^{t}$ or both have a low conductivity, the wall temperature fluctuations can persist and become a supplementary source of instability for the bulk, and then decreasing $R a_{c}$. The experimental values of $R a_{c}$ and $k_{c}$ found by Kebiche et al. [26] and Darbouli et al. [20] are much larger than those predicted by the linear theory when the finite thermal conductivity of the plates is taken into account. A possible explanation of this discrepancy is given in $\S 5$.

235 In Figure 2, the eigenfunctions, $F_{11}, G_{11}$ and $\tilde{G}_{11}$ are presented for different combinations of the conductivities of the upper and lower plates. Note that the symmetry of $F_{11}$ is little influenced by the thermal boundary conditions. 
As indicated above, the temperature fluctuation doesn't relax at the wall when $\xi^{t, b}<1$. With decreasing $\xi^{b, t}$, the fluctuation of the temperature gradient decreases. When, $\xi^{t, b}<<1, \partial \theta / \partial z \rightarrow 0$ and the situation of fixed heat flux at the boundary is recovered. Contours of the temperature perturbation are represented in Fig. 3 for three situations. The first one (Fig 3 a) represents the classical case where the boundaries are "good heat conductor". The second situation (Fig 3b) corresponds to the experimental device used by Kebiche et al. [27]. The horizontal plates are made of Polycarbonate with $\xi^{t}=\xi^{b}=0.25$. The third situation (Fig 3 ) corresponds to the experimental device used by Darbouli et al. [20] where $\xi^{t}=2$ and $\xi^{b}=201.6$. As we can see, the coupling between the temperature perturbation $\theta$ in the fluid and that in the horizontal plates $\tilde{\theta}$ is specific for each situation. With decreasing $\xi^{b, t}$, the horizontal scale of the convection motion increases and the vertical diffusion rate becomes greater than the horizontal one.

\subsection{Characteristic time of the instability}

Near the onset of convection, the growth rate $\mathcal{R} e(s)$ of the perturbation may be approximated using Taylor expansion,

$$
s=\frac{\epsilon}{\tau_{0}}+O\left(\epsilon^{2}\right) \quad \text { with } \quad \epsilon=\frac{R a-R a_{c}}{R a_{c}} .
$$



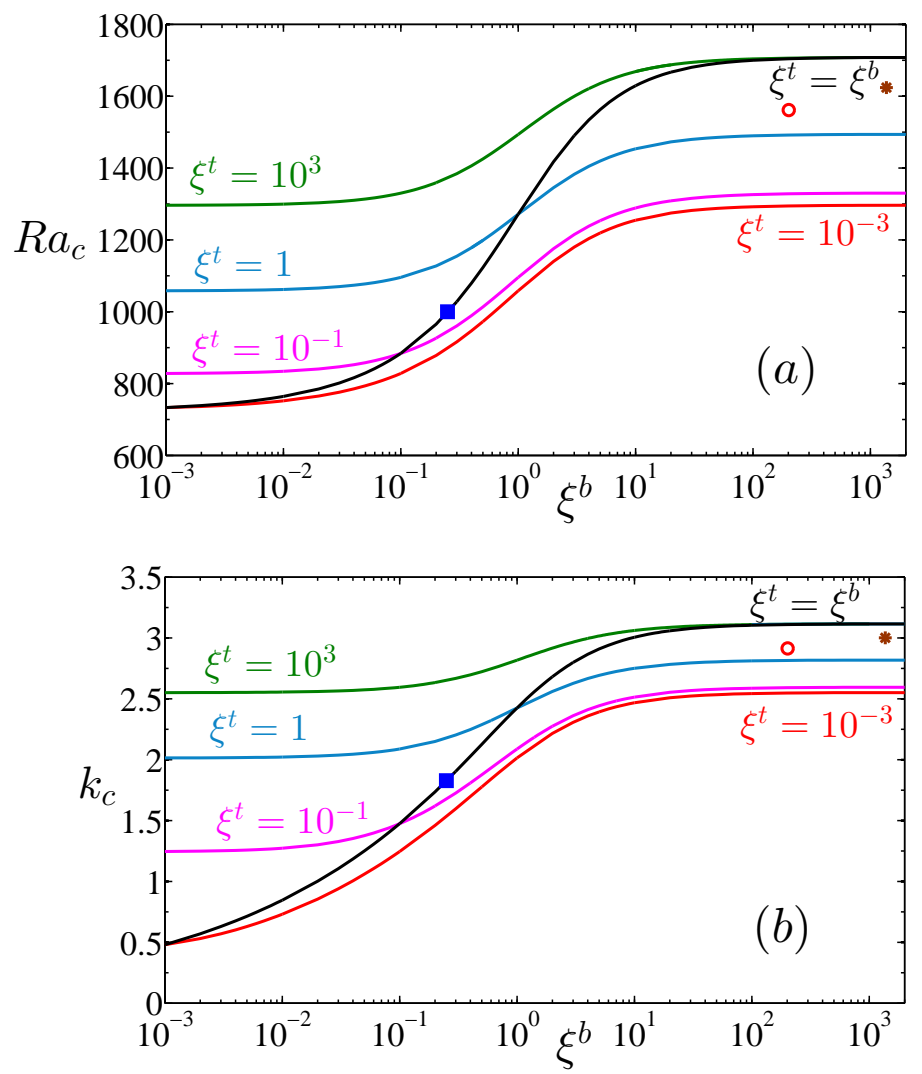

Figure 1: Critical Rayleigh number (a) and critical wavenumber (b) as function of $\xi^{b}$ for different values of $\xi^{t}$. The thick black line corresponds to the case $\xi^{t}=\xi^{b}$. The symbols are the theoretical values of $R a_{c}$ and $k_{c}$ corresponding to experimental devices used in the literature: (ם) Kebiche et al. 27], (०) Darbouli et al. 20] and (*) Hassan et al 28]. 

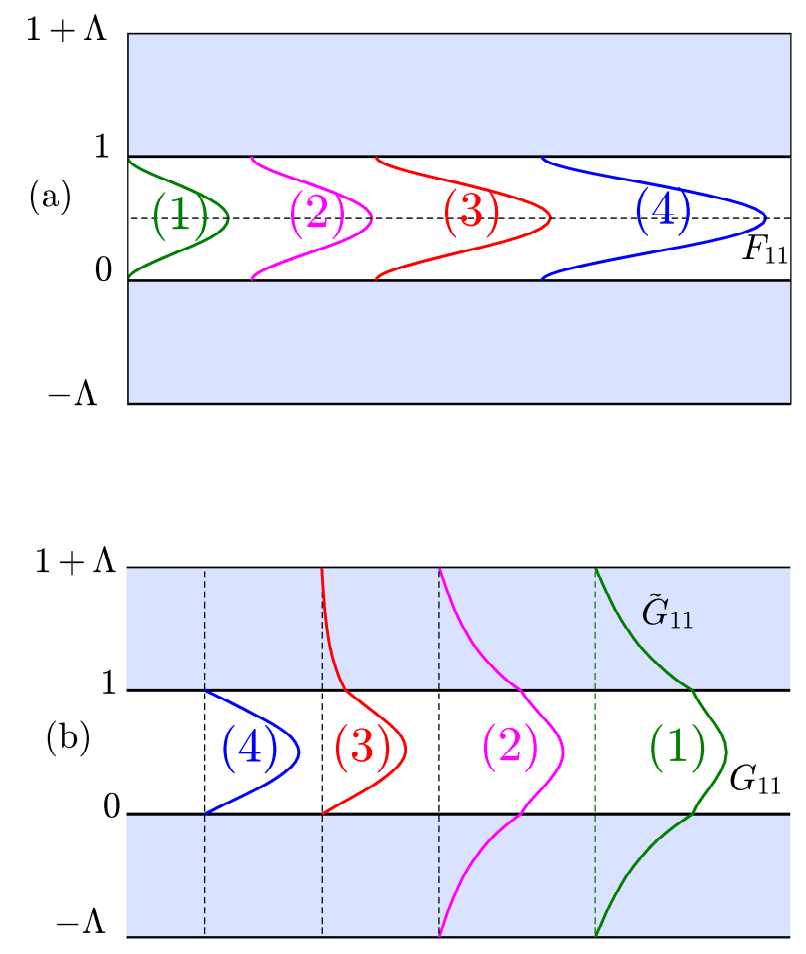

Figure 2: Eigenfunctions (a) $F_{11}$ (vertical velocity) and (b) $G_{11}, \tilde{G}_{11}$ (temperature perturbation) for different values of $\left(\xi^{b}, \xi^{t}\right)$ : (1) $\xi^{b}=\xi^{t}=0.1$; (2) $\xi^{b}=\xi^{t}=0.25$; (3) $\xi^{b}=201.6, \xi^{t}=2 ;(4) \xi^{b}=\xi^{t}=10^{3}$. 
(a)

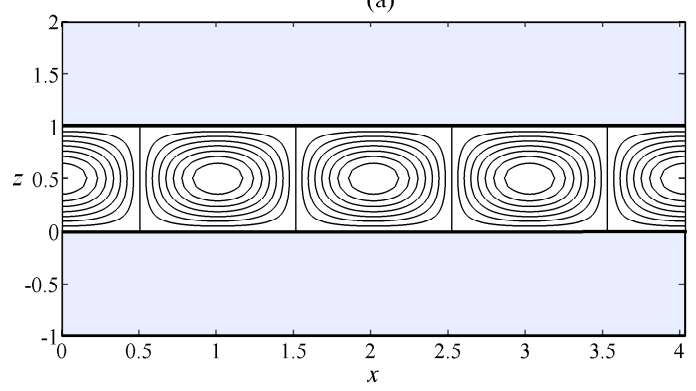

(b)

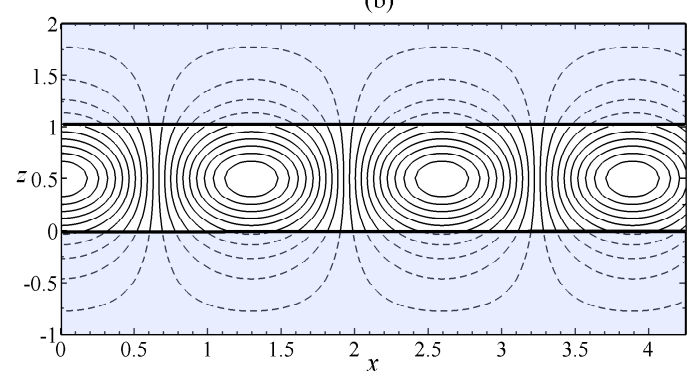

(c)

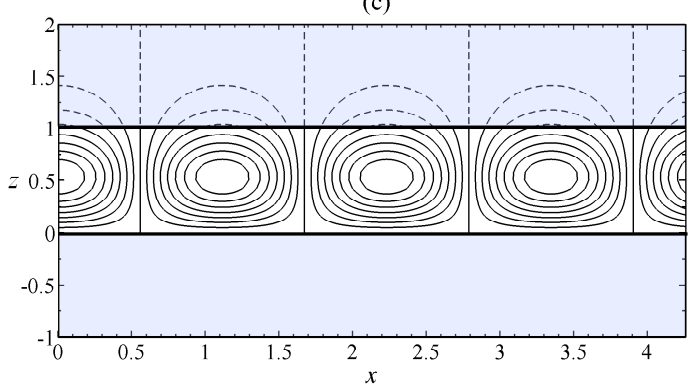

Figure 3: Temperature perturbation contours for three Rayleigh-Bénard situations : (a) $\xi^{b}=\xi^{t}=1000$ "good heat conductor", (b) $\xi^{b}=\xi^{t}=0.25$ corresponding to Kebiche et al. 27] experimental device and (c) $\xi^{b}=201.6 ; \xi^{t}=0.25$ corresponding to Darbouli et al. [20] experimental device. 


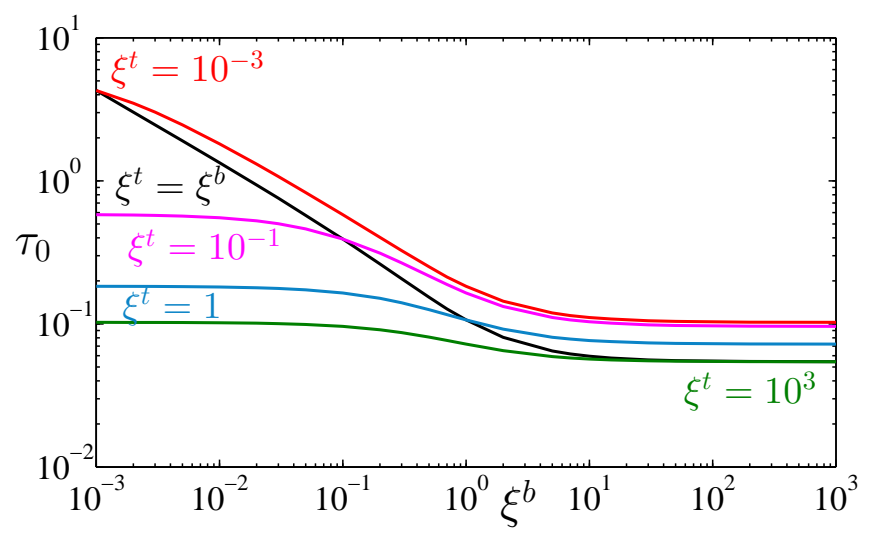

Figure 4: Characteristic time of instability vs thermal conductivities ratio $\xi^{b}$ at $\operatorname{Pr}=10$ in the case of $\xi^{t}=\xi^{b} ; \xi^{t}=10^{-3} ; \xi^{t}=10^{-1} ; \xi^{t}=1$ and $\xi^{t}=10^{3}$.

\section{Weakly nonlinear stability analysis: Pattern selection}

As it is known, linear stability analysis yields the onset of instability and the critical wave number. It is unable to give unique predictions for the form or evolution of the resulting patterns at a finite distance above the onset of convection [29]; this is so because the eigenvalue problem is degenerate, since to one eigenvalue $R a$ there corresponds an infinite number of possible patterns with the same wave number $k[30]$. The pattern selection is determined by the nonlinear terms. A weakly nonlinear analysis is adopted as a first approach to investigate nonlinear effects in the competition between rolls and squares [1], 17]. Hexagons configuration is not considered here. Further calculations show that this three-dimensional planform is unstable when the temperature dependence of the viscosity is not taken into account [1] [11] [18] [31].

Figure 5 shows, at critical conditions, the stability regions of square planforms in the $\left(\xi^{b}, \xi^{t}\right)$-space coordinate system at $\operatorname{Pr}=10$ for a Newtonian fluid and Carreau fluids with $n=0.5$ and different values of the dimensionless constant time $\lambda$. Squares are stable in the region bounded by the axis and the stability curve. Rolls are stable outside this region. In agreement with Riahi [18], stability curves are symmetric about the line $\xi^{b}=\xi^{t}$. 
The wide region, $\Phi$, corresponds to the Newtonian case and can be bounded approximately by the lines $\xi^{b}+\xi^{t} \leq 2$ and $\xi^{t}=\xi^{b}+0.7$. The ascertainment that squares are stable in this domain can be rationalized as follows. When the walls are poor heat conductors compared to the fluid, the convection intensity is low and the vertical velocity of the liquid $w$ is small [32]. So, this reflects a weak interaction between sets of rolls that constitute squares knowing that no two rolls predominates the other. As a consequence, only squares planforms are stable.

By introducing the shear-thinning character, the convection intensity increases 33] 34] 35] 36], thereby increasing the interaction between the two sets of rolls that constitute squares. As a consequence, one of the two sets of rolls nonlin295 early damps out the other and the squares become unstable contrary to rolls which become the preferred form of convection[1]. As illustrated in Fig [5] the region $\Phi$ decreases with increasing shear-thinning effects.

With increasing the reduced Rayleigh number $\epsilon=\left(R a-R a_{c}\right) / R a_{c}$, the nonlinear convection terms and non linear viscous terms become stronger, reducing the stability region of squares as illustrated in Figs. 6 and 7

These results are consistent with the maximum heat-transfer principle, "the only stable solution is the one of maximum heat transport" [37]. The Nusselt number is defined by

$$
N u=1-\left(\frac{\partial \bar{\theta}}{\partial z}\right)_{z=0} .
$$

The overbar denotes the horizontal average.

Rematk : Deviating from the critical conditions, $s>0$, the temperature profile in the plates, and the boundary conditions at the interface fluid/solid, involve a parameter denoted $\tilde{k}$ which depends on $s\left(\hat{\kappa}_{p}^{(b, t)} / \hat{\kappa}\right)$, see for instance Eqs. (32)-(34). For the computations at $\epsilon>0$, we have assumed as Chapman and 310 Proctor [23] and Carriere et al. [24] that $\hat{\kappa}_{p}^{(b, t)} / \hat{\kappa}=\hat{k}_{p}^{(b, t)} / \hat{k}$. This assumption can be justified by the fact that for for several couples fluid/plate, considered in Rayleigh-Bénard convection problems, the ratio of the thermal capacity of the 


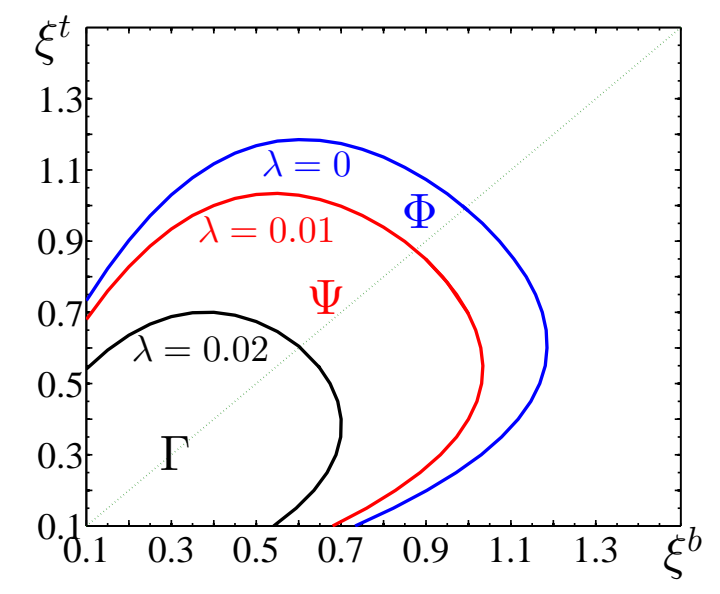

Figure 5: Stability boundary of squares in the $\left(\xi^{b}, \xi^{t}\right)$-space coordinate system at $\operatorname{Pr}=10$ for a Newtonian fluid $(\lambda=0)$ and Carreau fluids with $n=0.5$ two values of $\lambda$ : domain $\Psi$, $\lambda=0.01$ and domain $\Gamma, \lambda=0.02$.

fluid to that of the plate remains of order 1: $\left(\hat{\rho} \hat{C}_{p}\right)_{\text {fluid }} /\left(\hat{\rho} \hat{C}_{p}\right)_{\text {plate }}=O(1)$. Therefore, the ratio of the thermal diffusivity of the plate, $\hat{\kappa}_{p}$, to that of the fluid, $\hat{\kappa}$, is mainly governed by the thermal conductivities ratio $\xi$.

Figure 8 shows the variation of the Nusselt number as a function of $\epsilon$ for different thermal boundary considered in laboratory experiments. The largest Nusselt number is obtained for perfectly heat conducting slabs. For $\xi^{t}<<1$ and ${ }_{320} \xi^{b}<<1, \mathrm{Nu}$ is weakly larger for square-cell solution than for two-dimensional roll solution, but the difference is systematic. Shear-thinning effects reduce the viscous friction at the wall, which leads to an increase of the convection intensity and therefore of the Nusselt number as it is illustrated by Fig. 9. However, the enhancement of the heat transfer by shear-thinning effects is significantly 325 reduced for poorly heat conducting plates. 


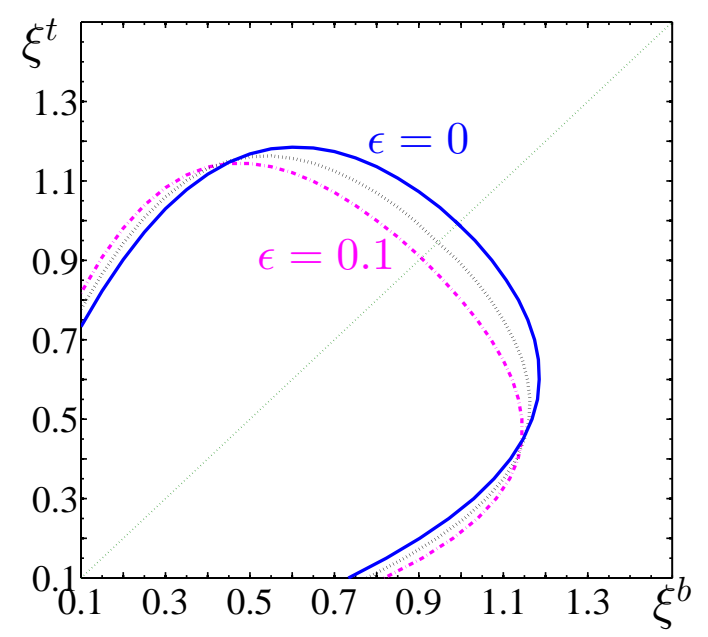

Figure 6: Stability boundary of squares in the $\left(\xi^{b}, \xi^{t}\right)$-space coordinate system for a Newtonian fluid at $\operatorname{Pr}=10$ and three different values of the reduced Rayleigh number $\epsilon$. (thick line) $\epsilon=0 ;$ (thin line) $\epsilon=0.05 ;$ (dotted line) $\epsilon=0.1$.

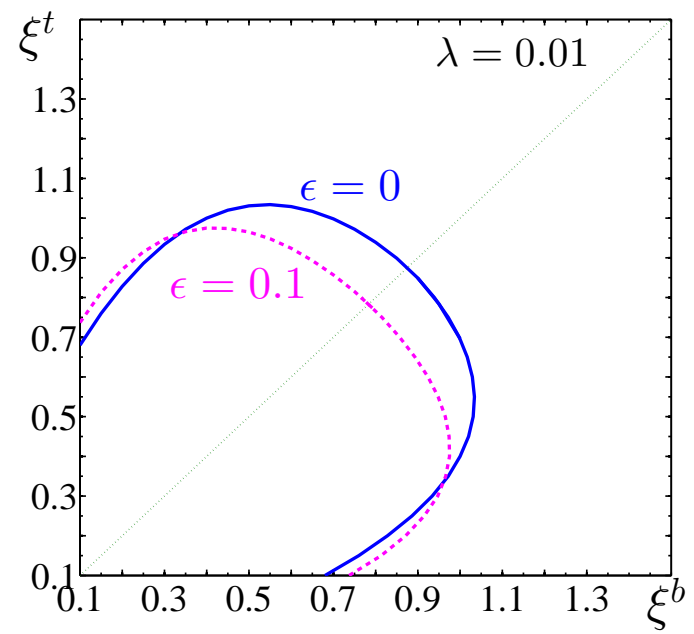

Figure 7: Stability boundary of squares in the $\left(\xi^{b}, \xi^{t}\right)$-space coordinate system for a Carreau fluid with $n=0.5$ and $\lambda=0.01$ at $\operatorname{Pr}=10$ and two different values of $\epsilon$. 


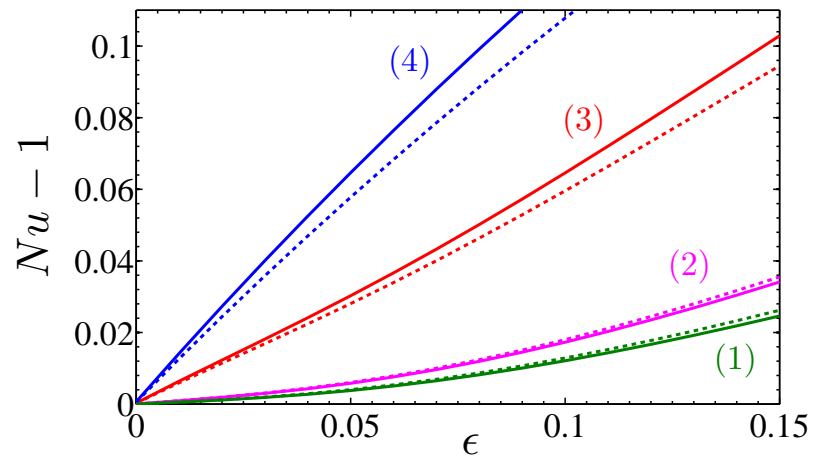

Figure 8: Nusselt number versus reduced the reduced Rayleigh number, $\epsilon$, for a Newtonian fluid with different thermal boundary conditions used in laboratory experiments: (1) $\xi^{t}=$ $\xi^{b}=0.25$ (Kebiche et al. 26]); (2) $\xi^{t}=\xi^{b}=0.4$ (LeGal and Croquette [15]; (3) $\xi^{t}=2$ and $\xi^{b}=201.6$ (Darbouli et al. 20]); (4) $\xi^{t}=\xi^{b}=10^{3}$. (dashed line): the planform convection is a square; (continuous line): the planform convection is a two dimensional roll.

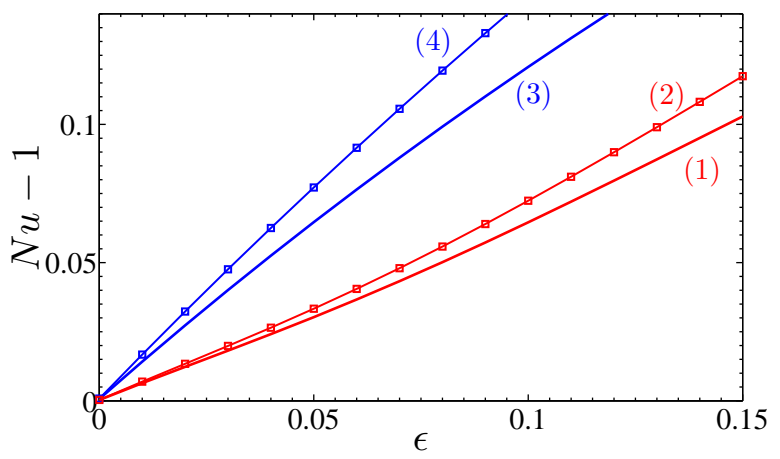

Figure 9: $N u$ versus $\epsilon$. Influence of shear-thinning effects for two different thermal boundary conditions: Curves (1) and (2) correspond to $\xi^{t}=2$ and $\xi^{b}=201.6$; curves (3) and (4) correspond to $\xi^{t}=\xi^{b}=1000$. Newtonian fluid: curves (1) and (2); Carreau fluid with $n=0.5, \lambda=0.01$, curves (2) and (4). 


\section{Conclusion}

In the present study we have investigated a linear and a weakly nonlinear stability analysis of a horizontal Carreau fluid layer bounded by two horizontal plates of arbitrary conductivity. Symmetry properties show that the the results are unchanged if the upper slab is replaced by the lower one and vice-versa.

The critical Rayleigh number and wavenumber associated to the onset of convection are determined as a function of the conductivities ratios $\xi^{t}$ and $\xi^{b}$. When either $\xi^{b}$ or $\xi^{t}$ or both are low, the wavelength of the convection becomes larger and the critical Rayleigh number $R a_{c}$ is reduced.

335 Stability analysis of the stationary solutions of the amplitude equations indicate that near the onset, the convection pattern is either a two-dimensional roll or a square. The influence of shear-thinning effects on the stability-domain of squares in the plane $\left(\xi^{t}, \xi^{b}\right)$ is highlighted. The results are in agreement with the maximum heat transfer principle.

340 The heat transfer and the convection intensity are strongly reduced when the plates are poor heat conductors. It is therefore more difficult to detect experimentally the onset of convection than in the case of 'good heat conductors'. This could explain the fact that $R a_{c}$ found by Kebiche et al. [26] and Darbouli et al. [20] is larger that that predicted by the linear theory.

Finally, we think that the present findings are useful to experimental observations since the finite conductivity of the walls plays a primordial role in the Rayleigh- Bénard convection and it can be a source of divergence between experimental observations and theoretical predictions.

Subsequent developments of the present work will concern the secondary instabilities of the primary pattern described here. Details on the secondary instabilities can be found in Ref. 38].

\section{Acknowledgments}

The authors gratefully acknowledge the financial support of the French National Research Agency, under the grant ANR-10-BLAN-925-01. 
[1] M. Bouteraa, C. Nouar, E. Plaut, C. Métivier, A. Kalck, Weakly nonlinear analysis of Rayleigh-Bénard convection in shear-thinning fluids: nature of the bifurcation and pattern selection, J. Fluid Mech. 767 (2015) 696-734.

[2] B. Holmedal, M. Tveitereid, E. Palm, Planform selection in RayleighBénard convection between finite slabs, J. Fluid Mech. 537 (2005) 255-270.

[3] R. Clever, F. H. Busse, Convection rolls and their instabilities in the presence of a nearly insulating upper boundary, J. Fluid Mech. 7 (1) (1995) $92-97$.

[4] D. B. White, The planforms and onset of convection with a temperaturedependent viscosity, J. Fluid Mech. 191 (1988) 247-286.

[5] E. Palm, On the tendency towards hexagonal cells in steady convection, J. Fluid Mech. 8 (02) (1960) 183-192.

[6] F. M. Richter, Experiments on the stability of convection rolls in fluids whose viscosity depends on temperature, J. Fluid Mech. 89 (03) (1978) 553-560.

[7] D. Oliver, J. Booker, Planform of convection with strongly temperaturedependent viscosity, Geophys. Astrophys Fluid Dyn 27 (1-2) (1983) 73-85.

[8] F. H. Busse, H. Frick, Square-pattern convection in fluids with strongly temperature-dependent viscosity, J. Fluid Mech. 150 (1985) 451-465.

[9] D. R. Jenkins, Rolls versus squares in thermal convection of fluids with temperature-dependent viscosity, J. Fluid Mech. 178 (1987) 491-506.

[10] L. Guillou, C. Jaupart, On the effect of continents on mantle convection, J. Geophys. Res. Solid Earth 100 (B12) (1995) 24217-24238.

[11] N. Riahi, Nonlinear convection in a porous layer with finite conducting boundaries, J. Fluid Mech. 129 (1983) 153-171. 
[12] F. H. Busse, N. Riahi, Nonlinear convection in a layer with nearly insulating boundaries, J. Fluid Mech. 96(02) (1980) 243-256.

[13] R. Proctor, Planform selection by finite amplitude thermal convection between poorly conducting slabs, J. Fluid Mech. 113 (1981) 469-485.

[14] D. R. Jenkins, M. R. E. Proctor, The transition from roll to square-cell solutions in Rayleigh-Bénard convection, J. Fluid Mech. 139 (1984) 461471 .

[15] P. Le Gal, A. Pocheau, V. Croquette, Square versus roll pattern at convective threshold, Phys. Rev. Lett. 54 (23) (1985) 2501.

[16] P. LeGal, V. Croquette, Appearance of a square pattern in a RayleighBénard experiment, Phys. Fluids 31(11) (1988) 3440-3442.

[17] M. Bouteraa, C. Nouar, Weakly nonlinear analysis of Rayleigh-Bénard convection in a non-Newtonian fluid between plates of finite conductivity: Influence of shear-thinning effects, Phys. Rev. E 92 (2015) 063017:1-13.

[18] N. Riahi, Nonlinear thermal convection with finite conducting boundaries, J. Fluid Mech. 152 (1985) 113-123.

[19] R. M. Clever, F. H. Busse, Convection in a layer heated from below with a nearly insulating boundary, Phys. Rev. E. 57 (1998) 4198-4205.

[20] M. Darbouli, C. Métivier, J.-M. Piau, A. Magnin, A. Abdelali, RayleighBénard convection for viscoplastic fluids, Phys. Fluids 25 (2) (2013) 023101.

[21] R. B. Bird, R. Amstrong, O. Hassager, Dynamics of polymeric liquids, Wiley-Interscience, New York, 1987.

[22] R. Tanner, Engineering rheology, Oxford University Press, New York, 2000.

[23] E. Sparrow, R. Goldstein, V. Jonsson, Thermal instability in a horizontal fluid layer: effect of boundary conditions and a non-linear temperature profile, J. Fluid Mech. 18(04) (1964) 513-528. 
[24] P. Carriere, A. Bottaro, P. Metzener, Wavelength selection in RayleighBénard convection between horizontal boundaries of finite conductivity, Eur. J. Mech. B/Fluids 16 (1997) 483-508.

[25] P. Cerisier, S. Rahal, J. Cordonnier, G. Lebon, Thermal influence of boundaries on the onset of Rayleigh-Bénard convection, Int. J. Heat. Mass Transfer 41 (1998) 3309-3320.

[26] Z. Kebiche, Etude expérimentale de l'instabilité de Rayleigh-Bénard dans les fluides non-Newtoniens, Ph.D. thesis, Université de Nantes (2014).

${ }_{415}[27]$ Z. Kebiche, C. Castelain, T. Burghelea, Experimental investigation of the Rayleigh-Bénard convection in a yield stress fluids, J. Non-Newtonian Fluid Mech. 203 (2014) 9-23.

[28] M. Hassan, M. Pathak, M. K. Khan, Rayleigh-Bénard convection in herschel-bulkley fluid, J. Non-Newtonian Fluid. Mech.

[29] M. Heutmaker, P. Fraenkel, J. P. Gollub, Convection patterns: time evolution of the wave-vector field, Phys. Rev. Lett. 54 (13) (1985) 1369.

[30] J. C.-V. G. Lebon, D. Jou, Understanding Non-equilibrium Thermodynamics, Springer Berlin Heidelberg, 2008.

[31] F. Busse, N. Riahi, Nonlinear convection in a layer with nearly insulating boundaries, J. Fluid. Mech 96 (02) (1980) 243-256.

[32] R. Verzicco, K. Sreenivasan, A comparison of turbulent thermal convection between conditions of constant temperature and constant heat flux, J. Fluid Mech. 595 (2008) 203-219.

[33] S. C. Pierre, C. Tien, Experimental investigation of natural convection heat transfer in confined space for non-Newtonian fluid, Can. J. Chem. Eng. 41 (1963) 122-127.

[34] S. F. Liang, A. Acrivos, Experiments on buoyancy-driven convection in non-Newtonian fluids, Rheol. Acta. 9 (1970) 447-455. 
[35] H. Ozoe, S. W. Churchill, Hydrodynamic stability and natural convection in Newtonian and non-Newtonian fluids heated from below, AIChE Symposium Series 69 (1973) 126-133.

[36] H.-S. Tsuei, C. Tien, Free convection heat transfer in a horizontal layer of non-Newtonian fluid, Can. J. Chem. Eng. 51 (2) (1973) 249-251.

[37] W. V. R. Malkus, G. Veronis, Finite amplitude cellular convection, J. Fluid Mech. 4 (1958) 225-260.

[38] R. Hoyle, Pattern formation. An introduction to methods, Cambridge University Press, 2006. 